\title{
Proactive Behavior of a Humanoid Robot in a Haptic Transportation Task with a Human Partner
}

\author{
Antoine Bussy $^{1} \quad$ Pierre Gergondet $^{1,2} \quad$ Abderrahmane Kheddar $^{1,2} \quad$ François Keith $^{1} \quad$ André Crosnier $^{1}$
}

\begin{abstract}
In this paper, we propose a control scheme that allows a humanoid robot to perform a complex transportation scenario jointly with a human partner. At first, the robot guesses the human partner's intentions to proactively participate to the task. In a second phase, the human-robot dyad switches roles: the robot takes over the leadership of the task to complete the scenario. During this last phase, the robot is remotely controlled with a joystick. The scenario is realized on a real HRP-2 humanoid robot to assess the overall approach.
\end{abstract}

\section{INTRODUCTION}

When two humans perform the transportation of an object together, such as a table, they are able to guess the other partner's intentions and act accordingly. The mutual understanding of each partner's intentions by the other generates proactive behaviors and good synchronization of the dyad during the task. Moreover, both partners may alternatively share the leadership of the task during its execution and take decisions such as turning or stopping, relying on the information they get. Because one might know and/or perceive something the other does not, a share of the leadership is desirable [1]. These are two characteristics we want to reproduce with a humanoid robot performing such a task with a human partner (see illustration on Fig. 1): proactivity and role switching.

Early works on physical Human-Robot Interaction (pHRI) gave the robot a passive role [2] where the human partner had to apply more forces than necessary in order to move the object due to the causality of the robot's control law. Its role was to carry a part of the object's vertical load at the cost of an increase of the horizontal 'load'. Proactivity aims at solving this problem: guessing the human partner's intentions to decrease this horizontal load. An approach is to regulate the robot's impedance according to the perceived intentions [3][4]. An other way to be proactive is to guess the human partner's intended trajectory. This is the approach chosen in [5][6] and also the one we choose in this paper. Note that both approaches are not incompatible.

Relatively to existing work, our approach distinguishes in its capability to guess the human partner's intentions for a wide variety of motions, where [5] and [6] only consider point-to-point movements. Furthermore, we distinguish the recognition of the partner's intended trajectory from the action undertaken to help him/her. Our proactive follower acts similarly to a leader. The difference is that it chooses

\footnotetext{
${ }^{1}$ Université Montpellier 2-CNRS, LIRMM, Interactive Digital Human group, 161 rue Ada, F-34095, Montpellier cedex 5, France. Contact: $<$ name $>$ @lirmm.fr

${ }^{2}$ CNRS-AIST Joint Robotics Laboratory UMI3218/CRT, Tsukuba, Japan.
}

to follow a trajectory determined from a guess of its partner's intentions rather than from its own volition. Thus our approach allows a natural role switching. We proposed a simpler one-degree-of-freedom control law based on a study performed with human subjects in [7] and this article generalizes it.

In Section II, we propose a compliant position control law for both leader and follower modes and how it can be used for role switching. We describe how a motion decomposition allows to recognize various intended trajectories in Section III. Because how a robot may behave as a leader is beyond the scope of this paper, we present how a human operator takes the control of the robot in the leader mode with a joystick in Section IV. We test our control scheme in Section V by making our HRP-2 humanoid robot perform the transportation scenario of Fig. 1 with a human partner.

\section{TRAJECTORY-BASED CONTROL LAW FOR PHRI}

\section{A. Notations and Hypothesis}

In this article, we are interested in a horizontal transportation task (two translation and one angular coordinates). We also want our robot to be compliant on the vertical axis. However, we first present our control law with only translations before introducing the rotation around the vertical axis at the end of this section. We therefore use a classical Cartesian world coordinate system for trajectories $\mathbf{X}$ and forces $\mathbf{F}$

$$
\mathbf{X}=\left(\begin{array}{c}
x \\
y \\
z
\end{array}\right) \quad \mathbf{F}=\left(\begin{array}{c}
f_{x} \\
f_{y} \\
f_{z}
\end{array}\right)
$$

As the transported object is supposed rigid and we are only considering translations, every point of the object has the same trajectory. Besides the robot is handling the object firmly without slipping, so every point of the robot's hands follows the same trajectory as the object. Thus we use the middle position of the hands to describe the object's trajectory and note it $\mathbf{X}$. Forces are calculated at this point. This choice is discussed further in Section III.

In this case, the object has the simple following dynamics

$$
M(\ddot{\mathbf{X}}-\mathbf{G})=\mathbf{F}
$$

where $M$ is the object inertia matrix, $\mathbf{G}$ the gravity vector and $\mathbf{F}$ the resultant of the forces applied on the object.

Unless sspecified otherwise, all variables are timedependent. 

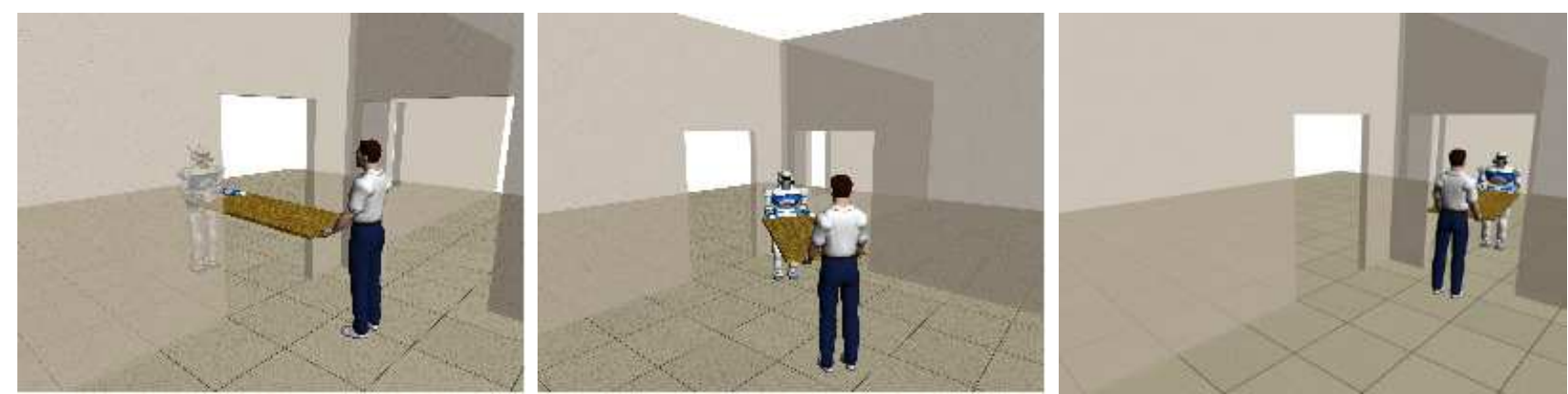

Fig. 1. Scenario of the experiment. The human-robot dyad has to carry the table through two doors that form a $90^{\circ}$ angle. The dimensions of the table are too big to perform the task with a single bend, so that the human has to pass backward through the first door and forward through the second one. The human assumes the leadership of the task as he is walking backward through the first door, and then is guided by the robot through the second door. During this second phase, the robot is remotely controlled by a second human thanks to a joystick.

\section{B. Proposed Control Law}

In this section, our goal is to control the Cartesian position of a supposedly rigid object, while maintaining a safe physical interaction with the human partner. Based on the equilibrium trajectory hypothesis [8], we propose the following simple trajectory-referenced admittance control law [9]:

$$
\mathbf{F}=-B \dot{\mathbf{X}}+K\left(\mathbf{X}_{0}-\mathbf{X}\right)
$$

where:

- $\mathbf{X}_{0}$ is the input equilibrium trajectory,

- $\mathbf{F}$ is the force applied by the manipulator,

- $B$ and $K$ are constant diagonal damping and stiffness matrices.

The problem is to determine the trajectory $\mathbf{X}_{0}$ that realizes accurate position control, while keeping the damping $B$ and stiffness $K$ at human-like levels. Given a twice continuously differentiable desired trajectory $\mathbf{X}_{d}$, the object inertia matrix $M$ and the gravity vector $\mathbf{G}$, we choose

$$
\mathbf{X}_{0}=K^{-1}\left[M\left(\ddot{\mathbf{X}}_{d}-\mathbf{G}\right)+B \dot{\mathbf{X}}_{d}+K \mathbf{X}_{d}\right] .
$$

In the case of a standalone manipulation, the simple object dynamic equation (2) becomes, with (3) and (4)

$$
M\left(\ddot{\mathbf{X}}-\ddot{\mathbf{X}}_{d}\right)+B\left(\dot{\mathbf{X}}-\dot{\mathbf{X}}_{d}\right)+K\left(\mathbf{X}-\mathbf{X}_{d}\right)=0
$$

whose solution $\mathbf{X}$ converges asymptotically to $\mathbf{X}_{d}$ with stable gains $M, B$ and $K$ : with no perturbation, the object follows the trajectory $\mathbf{X}_{d}$. The admittance control law (3) becomes

$$
\mathbf{F}=M\left(\ddot{\mathbf{X}}_{d}-\mathbf{G}\right)+B\left(\dot{\mathbf{X}}_{d}-\dot{\mathbf{X}}\right)+K\left(\mathbf{X}_{d}-\mathbf{X}\right)
$$

Equation (5) shows that if we can correctly predict the dynamics of the object, i.e. its inertia and all the forces exerted on it, we can adapt the equilibrium trajectory $\mathbf{X}_{0}$ so that the desired trajectory $\mathbf{X}_{d}$ is reached. In our simple case, it only means estimating the object inertia matrix $M$. It can easily be estimated at the experiment start-up by measuring the force vertical component. Note that an error of the dynamics prediction results in an error in $\mathbf{X}_{0}$ which is "filtered" by the admittance the same way as an error on $\mathbf{X}$ is.

\section{Behavior in Collaborative Mode}

Here, we assume that the forces applied by the other partner in collaborative mode cannot be predicted. Thus, the method used previously cannot be repeated. However, we show that there is an alternative method to achieve a desired trajectory. We also assume that both partners are able to share the load of the object dynamics. In our simple case, there is only the object predicted inertia and weight $M\left(\ddot{\mathbf{X}}_{d}-\mathbf{G}\right)$, i.e. the object mass. As the partners are sharing the object weight, they can undertake the inertia of the portion of the object they are carrying. In the following, we assume an equal sharing of $M / 2$ for simplicity. All the reasoning described in this subsection can be straightforwardly extended to several partners. The notations of the previous subsection are reused, indexed with the number of the partner $i \in\{1,2\}$.

Applying (3) and (4) for each partner, we obtain the following object dynamic equation

$$
M \ddot{\mathbf{X}}=\sum_{i=1}^{2} \frac{M}{2} \ddot{\mathbf{X}}_{d, i}+B_{i}\left(\dot{\mathbf{X}}_{d, i}-\dot{\mathbf{X}}\right)+K_{i}\left(\mathbf{X}_{d, i}-\mathbf{X}\right)
$$

For the sake of clarity, we assume that the human/robot control law is the one we propose, but it is only necessary for $\mathbf{X}_{d, 2}$ to be the solution of

$$
\frac{M}{2}(\ddot{\mathbf{X}}-\mathbf{G})=\mathbf{F}_{2}(\mathbf{X})
$$

i.e. the human/robot is able to perform accurately a desired trajectory when transporting half the object alone. In the case where

$$
\mathbf{X}_{d, 1}=\mathbf{X}_{d, 2}=\mathbf{X}_{d}
$$

the realized trajectory is $\mathbf{X}_{d}$ and $\mathbf{F}_{1}=\mathbf{F}_{2}=M \ddot{\mathbf{X}}_{d} / 2$ which is the equal sharing of the task. In practice, we would have

$$
\left\{\begin{array}{l}
\dot{\mathbf{X}}_{d, 1}=\dot{\mathbf{X}}_{d, 2}=\dot{\mathbf{X}}_{d} \\
\mathbf{X}_{d}=\left(K_{1}+K_{2}\right)^{-1}\left[K_{1} \mathbf{X}_{d, 1}+K_{2} \mathbf{X}_{d, 2}\right]
\end{array}\right.
$$

The position offset between $\mathbf{X}_{d, 1}$ and $\mathbf{X}_{d, 2}$ results in a cocontraction force between partners which is observed in [10].

Thus, in order to achieve an equal sharing of the task, both partners must have the same desired trajectory [1]. Otherwise, different desired trajectories results in internal 
(i.e. not working) forces exerted by the two partners. These statements are already well-known and predicting a human partner's desired trajectory is one of the main challenges in the pHRI field. However, how $\mathbf{X}_{d}$ is determined is completely independent of our control law, so that it can be used in both standalone and collaborative modes (leader and follower). The difference between these modes lies in the trajectory planning of $\mathbf{X}_{d}$. For a proactive follower behavior, $\mathbf{X}_{d}$ must be planned to match the human partner's intentions at best.

Besides, assuming we have trajectory planners for each of the three modes (standalone, leader, follower), it is possible to switch the robot behavior as theorized in [1][11] by switching the planners, without changing the control law that regulates the physical interaction.

\section{Rotation around the vertical axis}

If we consider a rotation around the vertical axis in our control law, the object dynamic equations become more complex with terms that depend on its geometry, even in the translational part. Predicting and compensating it is not as simple. We decide not to compensate the object dynamics that are introduced by the additional rotation, and to let them act as a perturbation on our control law.

\section{Proactive Trajectory Planner}

To be proactive, the robot first needs to correctly guess the human partner's intentions, and thus to locally predict his/her intended actions or trajectories. Motion prediction of the human partner has been addressed throughout the literature in pHRI. The strategy generally aims at reducing the problem to the estimation of handful parameters that allows generating a complete motion. The most famous example is the minimum jerk model [5][6]. However this model is always rather applied to point-to-point motion and does not fit for motions going beyond the reach of the arm or even to motion for which the target point is not well defined. When two humans perform a transportation task of an object, they might talk to give each other indications, such as "turn left", "go forward" or "stop". Based on this observation, we suggest to decompose the motion in phases, as it had been addressed for handshaking [4] and dancing [12].

The purpose of this part is to generate a plan for the robot in the form of a desired trajectory $\mathbf{X}_{d}$ that matches the human partner's intentions.

\section{A. Motion Primitives}

We decompose the motion into template sub-motions, or motion primitives, pictured in Fig. 2:

- Stop: no motion;

- Walk: walk forward or backward;

- Side: walk sideways;

- Turn: turn on itself;

- Walk/Turn: turn while walking forward or backward.

Sequencing these primitives allows to generate various motions, as in Fig. 3, while preventing some unnatural motions like walking in diagonal, i.e. Walk/Side. Moreover, we do not allow every sequence. For instance, Side cannot

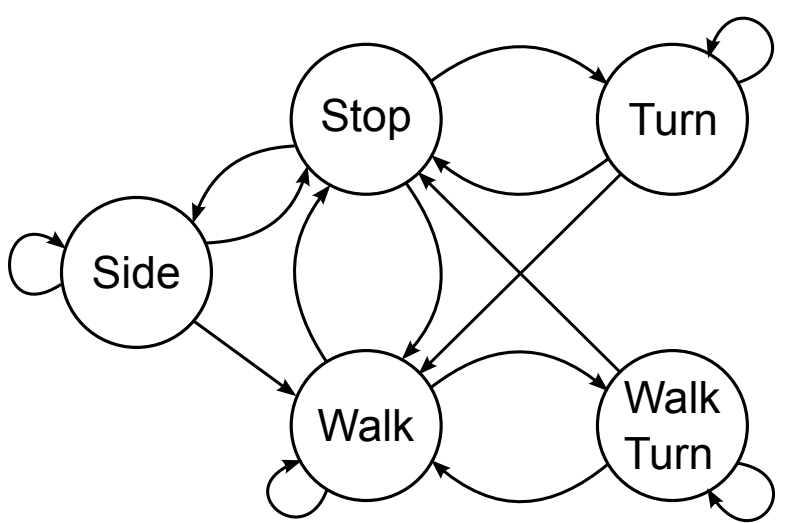

Fig. 2. Finite State Machine describing the possible primitives sequencing. It can generate sequences for both leader and follower modes. The transitions are triggered differently depending on the chosen mode.

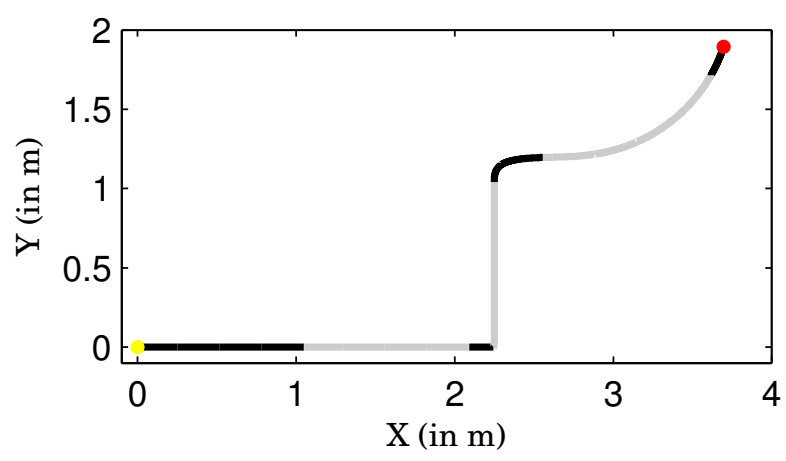

Fig. 3. Example of desired trajectory from yellow dot to red dot. The sequence of primitives is Stop, Walk, Walk(with a different $\mathscr{V}$ ), Stop, Side, Walk, Walk/Turn, Stop. The alternation of black and gray pictures the alternation of primitives. The sequence of $\mathscr{V}$ used is given by Table I.

follow Walk: the robot must stop walking before moving sideways. Each primitive is associated with a three dimension velocity vector $\mathscr{V}$ in a local frame ${ }^{1}$ (frontal, lateral and angular velocities) which is updated at each transition. As it can be seen from Table I, the signal $\mathscr{V}$ is piecewise constant over time and therefore does not represent a feasible trajectory. It should rather be considered as a simplified velocity plan, i.e. a template.

The local desired velocity $\mathbf{V}_{d, l}$ is generated from this plan by using a critically damped second order filter

$$
\frac{\mathbf{V}_{d, l}}{\mathscr{V}}=\frac{\omega_{0}^{2}}{\left(s+\omega_{0}\right)^{2}}
$$

where $\omega_{0}$ characterizes the rise time of the desired trajectory. For example, when transiting from Stop to Walk, the value of the first component of $\mathscr{V}$ instantly switches from 0 to $0.5 \mathrm{~m} / \mathrm{s}$. This velocity step need to be smoothed into a more human-like motion with filter (11).

Then, a change of frame is performed on $\mathbf{V}_{d, l}$ to get the desired velocity $\mathbf{V}_{d}$ in the global frame ( $x, y$,yaw). As we only consider planar motions, the vertical component, as well as the roll and pitch ones, are set to zero to obtain a six

\footnotetext{
${ }^{1}$ This frame has the same orientation as the robot but has a fixed origin in the world frame.
} 
TABLE I

EXAMPLE OF PRIMITIVE AND $\mathscr{V}$ SEQUENCES.

\begin{tabular}{|c||c|}
\hline Primitive & $\mathscr{V}$ \\
\hline \hline Stop & $(0.0,0.0,0.0)$ \\
\hline Walk & $(0.5,0.0,0.0)$ \\
\hline Walk & $(0.4,0.0,0.0)$ \\
\hline Stop & $(0.0,0.0,0.0)$ \\
\hline Side & $(0.0,0.4,0.0)$ \\
\hline Walk & $(0.5,0.0,0.0)$ \\
\hline Walk/Turn & $(0.5,0.0,0.5)$ \\
\hline Stop & $(0.0,0.0,0.0)$ \\
\hline
\end{tabular}

components vector. Finally, $\mathbf{V}_{d}$ is integrated into the desired trajectory $\mathbf{X}_{d}$ in the global frame.

\section{B. Turning}

We parametrize the turning motion with an angular velocity and omit the position of the center of rotation. Therefore the robot is only able to turn around the center of its hands because of our choice of $\mathbf{X}$ in Section II. When collaborating with direct contact (the human partner is directly holding the robot's hands) or in standalone mode, it is a reasonable choice and it has the advantage not to rely on the object's geometry. However, when carrying a table, the choice of the center of rotation depends on the motion to perform. For instance, when only rotating the table (primitive Turn), the center of the object is the most reasonable choice if we wish to minimize the distance both partners have to travel.

Putting the center of rotation too far away from the robot's body also forces the robot to perform lateral steps which puts a lot of strain on it. We therefore keep to our choice of Section II and leave the dynamic determination of a center of rotation to future work. The drawback is that it forces the human partner to travel much more distance than the robot when rotating the object on the spot.

\section{Reactive Generation of Primitives Sequences}

In our approach, predicting the leader's intended trajectory consists in determining a primitives sequence that matches it. We mainly use velocity thresholds to detect the switches of primitives. For example, when the current primitive is Stop and the effective velocity $\mathbf{V}$ of the object is zero, the robot senses a force on its wrists and updates $\mathbf{V}$ with (3). If the first component of $\mathbf{V}$ exceeds a given threshold, the robot switches to the primitive Walk.

We use velocity thresholds instead of force thresholds because of the co-contraction force between the partners. This co-contraction force may vary between dyads and trials, so that good force thresholds cannot be found. There is no such problem with the velocity because it is partly the high-pass filtered force (3). Nevertheless, the leader might indefinitely increase the force very slowly without triggering the velocity thresholds. To avoid such a situation, we also add high force thresholds, which are tuned to be less reactive than the velocity ones.

Self-transitions are also regularly triggered to update $\mathscr{V}$, e.g. every second, with the current velocity $\mathbf{V}$ of the object, so that the robot is able to adapt its desired velocity. The subsequence Walk, Walk(with a different $\mathscr{V}$ ) of Fig. 3 is an example of self-transition. When switching from a primitive to a different one, i.e. not a self-transition, we set $\mathscr{V}$ to a fixed default value and let it be updated at the next self-transition one second later.

\section{Switch to Leader Mode with a Joystick}

As stated in Section II, our pHRI control law is independent of how the desired trajectory $\mathbf{X}_{d}$ is generated and thus allows easy role switching between follower and leader behaviors. To demonstrate the capability of our control scheme to do so, we generates an intended trajectory $\mathbf{X}_{d}$ for the robot from a joystick. Thus a second human can pilot the robot during the task of transporting the table with the first human partner.

We use a joystick with a digital directional touchpad to control the robot in leader mode. We use the same FSM as in the follower mode (Fig. 2), where the transitions are triggered by the touchpad state instead of haptic clues, thus determining the motion direction. The velocity amplitude is set constant and not controlled by the joystick. The output plan $\mathscr{V}$ from the FSM is then used the same way it is in Section III to compute the desired trajectory $\mathbf{X}_{d}$ for the impedance control. The joystick operator can assume or give up the leadership of the task by pressing a specific key on the joystick. The minimal input we use from the joystick and the unnecessary force feedback assess the robustness of our control scheme.

\section{EXPERIMENTATION ON THE HRP-2 HUMANOID Rовот}

\section{A. Scenario}

To validate our proposed control scheme, we realize the scenario described in Fig. 1.

\section{B. Whole Body Motion and Walking}

The HRP-2 humanoid robot interacts with its environment through two force-torque sensors mounted on each wrist that measure two forces $\mathbf{F}_{L}$ and $\mathbf{F}_{R}$, that we transport at point $\mathbf{X}$ and sum to get the force feedback $\mathbf{F}$ for the admittance controller. The stiffness $K$ and damping $B$ diagonal coefficients are experimentally tuned (Table II). The admittance controller output $\mathbf{X}$ is used to position-control the hands through the Stack-of-Tasks (SoT) developed in [13], a generalized inverted kinematics. The SoT allows to define various tasks -positioning the hands in the world frame in our case- and uses the robot redundancy to realize them simultaneously.

TABLE II

STIFFNESS AND DAMPING COEFFICIENTS.

\begin{tabular}{|c||c|}
\hline Stiffness & Damping \\
\hline \hline$K_{x y}=40 \mathrm{~N} / \mathrm{m}$ & $B_{x y}=85 \mathrm{~N} . \mathrm{s} / \mathrm{m}$ \\
\hline$K_{z}=250 \mathrm{~N} / \mathrm{m}$ & $B_{z}=200 \mathrm{~N} . \mathrm{s} / \mathrm{m}$ \\
\hline$K_{\theta}=25 \mathrm{~N} . \mathrm{m} / \mathrm{rad}$ & $B_{\theta}=50 \mathrm{~N} . \mathrm{m} . \mathrm{s} / \mathrm{rad}$ \\
\hline
\end{tabular}


For the locomotion, we used a modified version of the walking Pattern-Generator (PG) developed in [14]. The PG generates on-line a trajectory for the Center of Mass (CoM) of the robot as well as trajectories for the feet, that are also executed through the SoT. These trajectories are calculated through a quadratic optimization with linear constraints, which is made possible by the use of a linearized model of the robot and a state-of-the-art quadratic problem solver. The PG minimizes, among others, the error between the CoM velocity and an input velocity, and the CoM jerk, with constraints on the robot's Zero Moment Point (ZMP) to insure its balance while walking.

The PG takes a 3D desired CoM velocity as an input: two translation and one angular velocities. We regulates this velocity input with a proportional controller so that the relative position of the robot's CoM and hands stays constant, as well as the relative orientation of its feet and hands. However, walking produces oscillatory motions by nature, especially on the lateral component, so we add deadzones so that the proportional controller does not compensate these oscillations. In the case of lateral motion, the deadzone produces an undesired static error: the robot's hands and body are visibly not aligned. We add an integrative term to align them; the integration is performed over a walk cycle.

\section{Results}

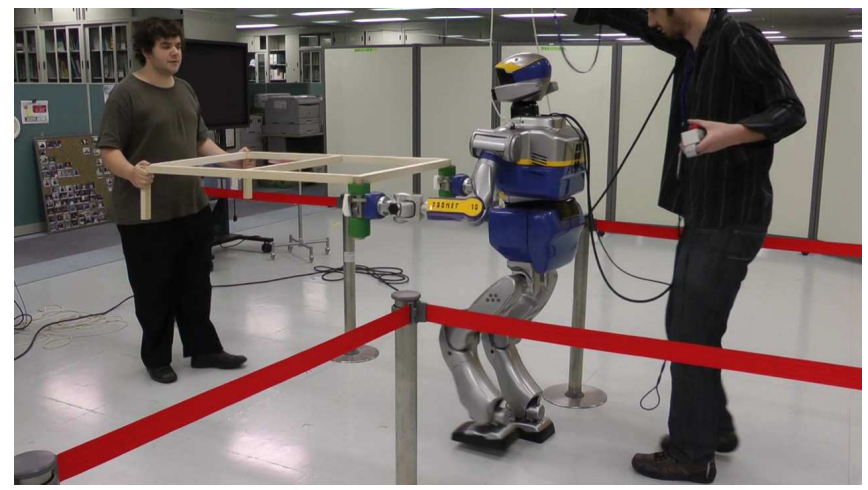

Fig. 4. HRP-2 realizing the transportation task with a human partner.

As pictured in Fig. 4 and in the accompanying video, our robot successfully performed the proposed scenario with a human partner. Trajectories $\mathbf{X}$ and $\mathbf{X}_{d}$ are shown in Fig. 5. Their corresponding velocities on the frontal axis - the direction of the motion - are shown in Fig. 6. Forces applied by the robot on the object on the frontal axis are shown in Fig. 7. We can observe that although the robot's plan $\mathbf{X}_{d}$ roughly approximates the object's effective trajectory, the force applied on object by the robot is greatly reduced compared to a fully passive behavior during the follower mode (until $t=20 \mathrm{~s}$ ). Note that during the follower mode, the robot applies negative mechanical power on the object. Around $t=12 \mathrm{~s}$ the robot wrongly detects an intention to stop from the leader, but is able to quickly recover and start off again. It results in a high peak in the force profile. Such a misunderstanding might also happen with a human/human dyad.

At around $t=20 \mathrm{~s}$, the joystick operator takes over the control on the robot and completes the scenario. And the human partner is able to follow the robot. The interesting point is that during the second part of the scenario, the force applied by the robot on the object and the velocity of the object have the same sign. The robot applies positive mechanical power on the object, and therefore the human partner applies negative power at constant velocity. Moreover, the leader phase's force intensity is similar to the follower phase's, which shows that our implementation of the robot's follower behavior yields similar results to the human partner's performance as a follower, force-wise at least.

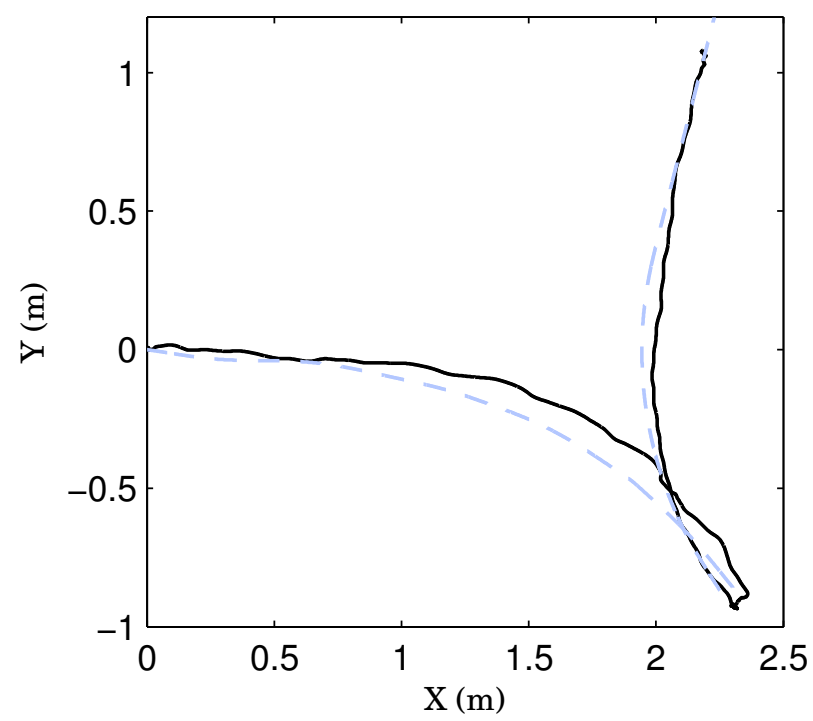

Fig. 5. Trajectories of the object in the XY plan: the admittance controller output $\mathbf{X}$ in black, follower desired trajectory $\mathbf{X}_{d}$ in dotted light blue. The transportation starts at $(0,0)$ and ends around $(2,1)$. The role switching occurs when the object is around $(2.3,-1)$.

\section{CONCLUDING REMARKS}

In this paper, we propose a complete control scheme that allows our HRP-2 robot to perform a transportation task with locomotion, jointly with a human partner.

The first main contribution is the ability of our control scheme to produce a proactive follower behavior. Thanks to a decomposition of the task in sub-motions, the robot is able to guess the human partner's intended trajectory that leads to a substantial reduction of the workless interaction force. Its amplitude is similar to the one observed when the human partner acts as a follower. Our results are similar to the ones obtained in [6] with a similar approach, but allows performing a wider variety of motions.

The second important contribution is the possibility to switch between the follower and leader behaviors in the course of the task. The next step is to find a method to reactively generate a leader plan for the robot and how and when to automatically switch between the two modes. 

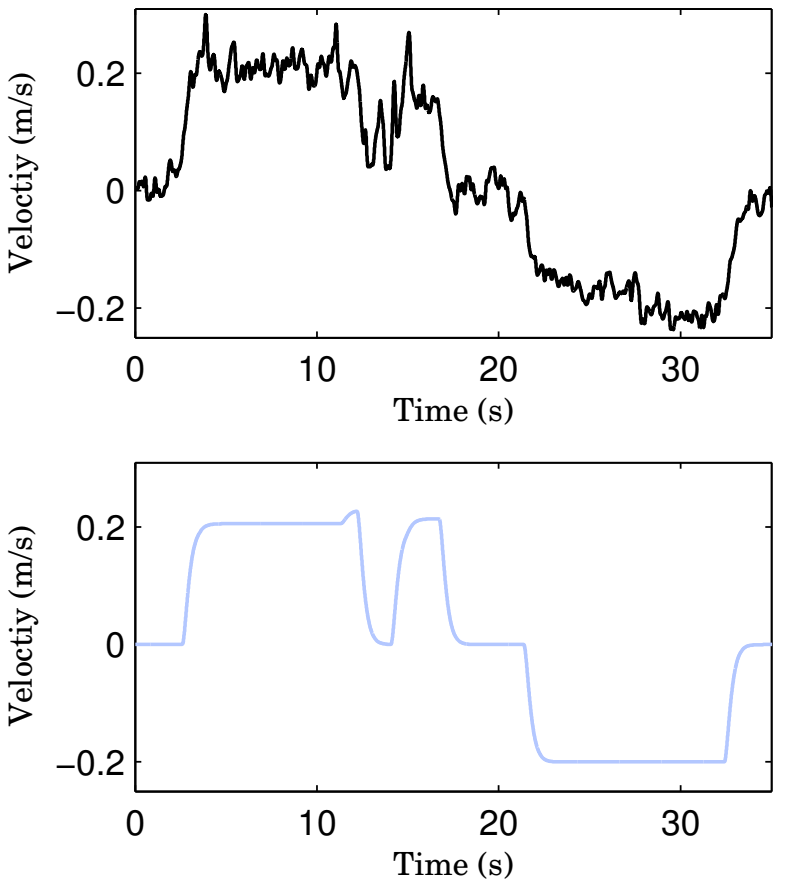

Fig. 6. Velocities of the object on the robot's frontal axis: the admittance controller output $\dot{\mathbf{X}}$ in black (top), robot desired velocity $\dot{\mathbf{X}}_{d}$ in light blue (bottom). The role switching occurs at around $t=20 \mathrm{~s}$.

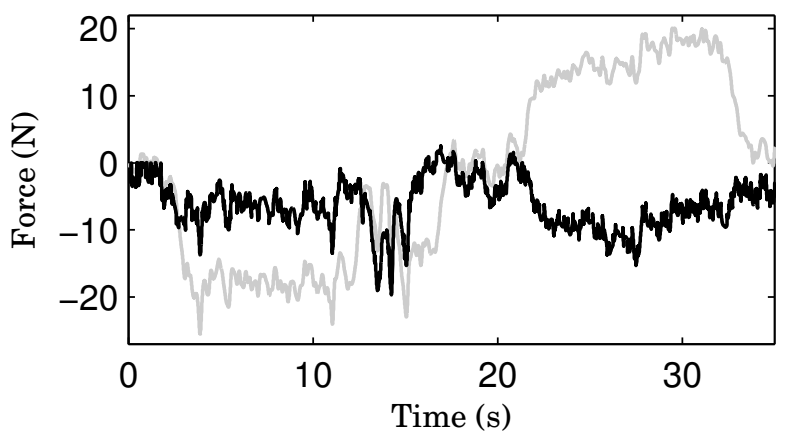

Fig. 7. Force applied by the robot on the object (black) on the robot's frontal axis. The gray curve represents the damping part of the interaction force $\mathbf{F}=-B \dot{\mathbf{X}}$ : it is the force that would be applied by the robot with a passive behavior. The role switching occurs at around $t=20 \mathrm{~s}$.

Our approach can be made more complex with additional primitives to widen the possible motions and tasks. We are thinking about primitives that allow precise positioning of the manipulated object, which we are not considering in our transportation task. It can also be generalized to completely different tasks if one can find a good decomposition of the task in elementary subtasks and a method to determine the switch timings between these subtasks.

We also uses constant impedance parameters. Coupling our approach with a variable impedance one as in [3] may improve the proactivity as well as the quality of the haptic feeling for the human partner.

As preliminary investigations for future work, we also tried our control scheme with an EEG-based BCI [15], i.e. a
BCI based on electroencephalography technology for signal acquisition. It roughly works as a poor joystick, but is usable by locked-in patients. Preliminary results show that BCIbased control is less reactive than the joystick and produces more lag as well as false positives (i.e. wrong choices of the desired direction), which is not compatible with our implementation of the FSM. Thus we directly use the BCI to determine the motion direction (forward/backward, rotate left/right) with fixed amplitude; in other words, the template plan $\mathscr{V}$. We shall improve this in our forthcoming work.

\section{ACKNOWLEDGMENT}

This work is supported in part by the FP7 IP RoboHow.Cog project (www.robohow.eu).

\section{REFERENCES}

[1] A. Kheddar, "Human-robot haptic joint actions is an equal controlsharing approach possible?" in IEEE International Conference on Human System Interactions (HSI), 19-21 May 2011, pp. 268-273.

[2] K. Kosuge, H. Yoshida, and T. Fukuda, "Dynamic control for robothuman collaboration," in Proceedings of the 2nd IEEE International Workshop on Robot and Human Communication. IEEE, 1993, pp. 389-401.

[3] V. Duchaine and C. M. Gosselin, "General model of human-robot cooperation using a novel velocity based variable impedance control," in Joint EuroHaptics Conference and Symposium on Haptic Interfaces for Virtual Environment and Teleoperator Systems. Washington, DC, USA: IEEE, 2007, pp. 446-451.

[4] Z. Wang, A. Peer, and M. Buss, "An hmm approach to realistic haptic human-robot interaction," in Joint EuroHaptics Conference and Symposium on Haptic Interfaces for Virtual Environment and Teleoperator Systems. IEEE, March 2009, pp. 374-379.

[5] Y. Maeda, T. Hara, and T. Arai, "Human-robot cooperative manipulation with motion estimation," in IEEE/RSJ International Conference on Robots and Intelligent Systems. IEEE/RSJ, October-November 2001, pp. 2240-2245.

[6] B. Corteville, E. Aertbelien, H. Bruyninckx, J. D. Schutter, and H. V. Brussel, "Human-inspired robot assistant for fast point-topoint movements," in IEEE International Conference on Robotics and Automation. IEEE, April 2007, pp. 3639-3644.

[7] A. Bussy, A. Kheddar, A. Crosnier, and F. Keith, "Human humanoid haptic joint object transportation case study," in IEEE/RSJ International Conference on Robots and Intelligent Systems, 2012.

[8] T. Flash, "The control of hand equilibrium trajectories in multi-joint arm movements," Biological Cybernetics, vol. 57, pp. 257-274, 1987

[9] N. Hogan, "Impedance control: an approach to manipulation," Journal of Dynamic Systems, Measurement, and Control, vol. 107, pp. 1-24, March 1985.

[10] K. B. Reed, "Understanding the haptic interactions of working together," Ph.D. dissertation, Northwestern University, Evanston, Illinois, USA, June 2007.

[11] P. Evrard and A. Kheddar, "Homotopy switching model for dyad haptic interaction in physical collaborative tasks," in Joint EuroHaptics Conference and Symposium on Haptic Interfaces for Virtual Environment and Teleoperator Systems. IEEE, March 2009.

[12] T. Takeda, Y. Hirata, and K. Kosuge, "Hmm-based error recovery of dance step selection for dance partner robot," in IEEE International Conference on Robotics and Automation. IEEE, April 2007, pp. 1768-1773.

[13] N. Mansard, O. Stasse, P. Evrard, and A. Kheddar, "A versatile generalized inverted kinematics implementation for collaborative humanoid robots: The stack of tasks," in IEEE Internationl Conference on Advanced Robotics, June 2009.

[14] A. Herdt, N. Perrin, and P.-B. Wieber, "Walking without thinking about it," in IEEE/RSJ International Conference on Intelligent Robots and Systems (IROS), 2010, pp. 190-195.

[15] P. Gergondet, S. Druon, A. Kheddar, C. Hintermüller, C. Guger, and M. Slater, "Using brain-computer interface to steer a humanoid robot," in IEEE International Conference on Robotics and Biomimetics, 2011, pp. 192-197. 